\title{
Two-Dimensional Critical Potts and its Tricritical Shadow
}

\author{
Wolfhard Janke \\ Institut für Theoretische Physik, Universität Leipzig, Augustusplatz, 10/11, 04109 Leipzig, Germany \\ and Adriaan M. J. Schakel \\ Institut für Theoretische Physik, Freie Universität Berlin, Arnimallee 14, 14195 Berlin, Germany
}

Received on 11 October, 2005

\begin{abstract}
These notes give examples of how suitably defined geometrical objects encode in their fractal structure thermal critical behavior. The emphasis is on the two-dimensional Potts model for which two types of spin clusters can be defined. Whereas the Fortuin-Kasteleyn clusters describe the standard critical behavior, the geometrical clusters describe the tricritical behavior that arises when including vacant sites in the pure Potts model. Other phase transitions that allow for a geometrical description discussed in these notes include the superfluid phase transition and Bose-Einstein condensation.
\end{abstract}

Keywords: Potts model; Tricritical behavior; Geometrical clusters; Fractal structure

\section{INTRODUCTION}

The quest for understanding phase transitions in terms of geometrical objects has a long history. One of the earlier examples, due to Onsager, concerns the superfluid phase transition in liquid ${ }^{4} \mathrm{He}$ - the so-called $\lambda$ transition. During the discussion of a paper presented by Gorter, Onsager [1] made the following remark: "As a possible interpretation of the $\lambda$ point, we can understand that when the concentration of vortices reaches the point where they form a connected tangle throughout the liquid, then the liquid becomes normal." Feynman also worked on this approach and summarized the idea as follows [2]: "The superfluid is pierced through and through with vortex line. We are describing the disorder of Helium I." This approach focuses on vortex loops, i.e., one-dimensional geometrical objects, which form a fluctuating vortex tangle. As the critical temperature $T_{\lambda}$ is approached from below, the vortex loops proliferate and thereby disorder the superfluid state, causing the system to revert to the normal state. The $\lambda$ transition is thus characterized by a fundamental change in the typical vortex loop size. Whereas in the superfluid phase only a few small loops are present, close to $T_{\lambda}$ loops of all sizes appear. The sudden appearance of arbitrarily large geometrical objects is reminiscent of what happens in percolation phenomena at the percolation threshold where clusters proliferate. Even on an infinite lattice, a percolating cluster can be found spanning the lattice.

A second example, due to Feynman [3], is related to BoseEinstein condensation. Here, the relevant geometrical objects are worldlines. In the imaginary-time formalism, used to describe quantum systems at finite temperature $T$, the time dimension becomes compactified, $t=-\mathrm{i} \tau$, with $0 \leq \tau \leq \hbar / k_{\mathrm{B}} T$, where $k_{\mathrm{B}}$ is Boltzmann's constant. Because of periodic boundary conditions, the worldlines then form closed loops. At high temperatures, where the system behaves more or less classically, the individual particles form separate closed loops wrapping only once around the imaginary time axis. Upon lowering the temperature, these small loops, describing single particles, hook up to form larger exchange rings. A particle in such a composite ring (see Fig. 1) moves in imaginary time along a trajectory that does not end at its own starting position, but ends at that of another particle. Hence, although the initial and final configurations are identical, the particles in a composite ring are cyclically permuted and thus become indistinguishable [3]. Fig. 1 gives an example of three particles, labeled 1,2, and 3. After wrapping once around the imaginary time axis particle 1 ends at the starting position of particle 2 , which in turn ends after one turn around the imaginary time axis at the starting position of particle 3 . That particle, finally, ends at the starting position of particle 1 . In this way, the three particles are cyclically permuted, forming the cycle $(1,2,3)$. Being part of a single loop which winds three times around the imaginary time axis, the particles cannot be distinguished any longer. At the critical temperature, worldlines proliferate and-again as in percolation phenomena-loops wrapping arbitrary many times around the imaginary time axis appear, signaling the onset of Bose-Einstein condensation [4, 5]. This

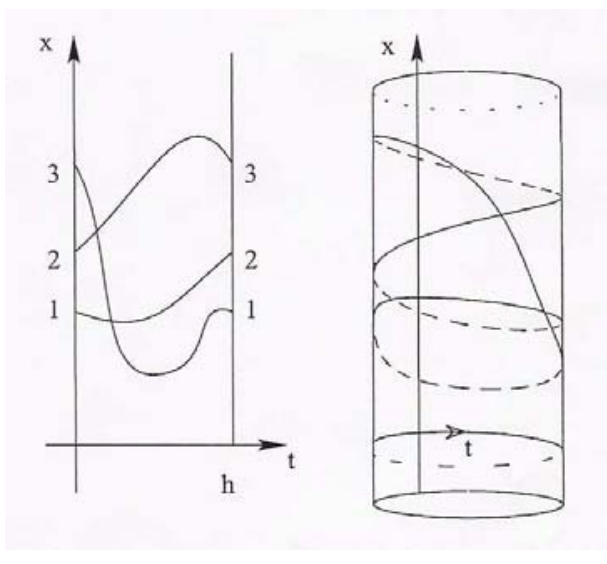

FIG. 1: The worldlines of three particles that, after moving a time $\tau=\hbar / k_{\mathrm{B}} T$ in the imaginary time direction, are cyclically permuted (left panel). The three separate worldlines can also be represented by a single worldline that winds three times around the imaginary time axis (right panel). (After Ref. [4].) 

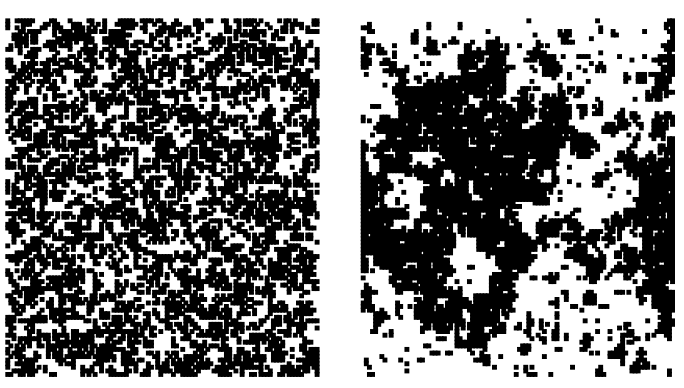

FIG. 2: Snapshots of typical spin configurations of the Ising model on a square lattice of linear size $L=100$ in the normal, hot phase at $\beta=0.5 \beta_{\mathrm{c}}$ (left panel) and just above the Curie point at $\beta=0.98 \beta_{\mathrm{c}}$ (right panel). A spin up is denoted by a black square, while a spin down is denoted by a white one.

approach has been turned into a powerful Monte Carlo method by Ceperley and Pollock [6] that can even handle strongly interacting systems like superfluid ${ }^{4} \mathrm{He}$ (see Ref. [7] for a review).

A third example concerns the phase transition in simple magnets. The most elementary model describing such a transition is provided by the Ising model, obtained by assigning a spin that can point either up or down to each lattice site. Fig. 2 shows typical spin configurations for a square lattice in the normal, hot phase and just above the Curie point. For convenience, a spin up is denoted by a black square, while a spin down is denoted by a white one. From these snapshots, the relevant geometrical objects appear to be clusters of nearest neighbor spins in the same spin state (in the following, we will qualify this statement). The normal, disordered phase consists of many small clusters. As the Curie point $T_{\mathrm{c}}$ is approached from above, larger clusters appear, which at $T_{\mathrm{c}}$ start to proliferate-as in percolation phenomena. In the absence of an applied magnetic field, the percolating cluster can consist of either up or down spins, both having equal probability to form the majority spin state. Since the percolating spin clusters have a fractal structure, it is tempting to ask whether this structure encodes the standard thermodynamic critical behavior, as in percolation theory? More generally, we wish to address in these notes the question: Can suitably defined geometrical objects encode in their fractal structure the standard critical behavior of the system under consideration? To highlight the basic features, we consider simple models, such as the Ising, the Potts, and the XY model. Moreover, we study them mostly in two dimensions (2D) since many analytical predictions, obtained by using Coulomb gas methods and conformal field theory, are available there.

The rest of these notes is organized as follows. In the next section, the 2D critical Potts model is discussed. Central to the discussion is the equivalent geometrical representation of this spin model in terms of so-called Fortuin-Kasteleyn clusters [8]. The fractal structure of these stochastic clusters and the way the thermal critical behavior of the Potts model can be extracted from it are studied in detail. In Sec. III, the tricritical Potts model is discussed. The clusters encoding the tricritical behavior turn out to be the naive clusters of nearest neighbor spins in the same spin state, which feature in Fig. 2. Their fractal structure is connected via a dual map to that of the Fortuin-Kasteleyn clusters, which encode the thermodynamic critical behavior. In Sec. IV, the boundaries of both cluster types are studied. The notes end with a summary of the main results and an outlook to other applications.

\section{CRITICAL POTTS MODEL}

\section{A. Fortuin-Kasteleyn Representation}

The Potts model is one of the well studied spin models in statistical physics [9]. It is defined by considering a lattice with each lattice site given a spin variable $s_{i}=1,2, \cdots, Q$ that can take $Q$ different values. In its standard form, the spins interact only with their nearest neighbors specified by the Hamiltonian

$$
\mathcal{H}=-K \sum_{\langle i j\rangle}\left(\delta_{s_{i}, s_{j}}-1\right)
$$

where $K$ denotes the coupling constant. Nearest neighbor spins notice each other only when both are in the same spin state, as indicated by the Kronecker delta. The Potts model is of particular interest to us as for $Q=2$ it is equivalent to the Ising model, while in the limit $Q \rightarrow 1$ it describes ordinary, uncorrelated percolation. The notation $\sum_{\langle i j\rangle}$ is to indicate that the double sum over the lattice sites, labeled by $i$ and $j$, extends over nearest neighbors only. The partition function $Z$ can be written as

$$
Z=\operatorname{Tr} \mathrm{e}^{-\beta \mathcal{H}}=\operatorname{Tr} \prod_{\langle i j\rangle}\left[(1-p)+p \delta_{s_{i}, s_{j}}\right]
$$

where $\beta$ denotes the inverse temperature, and the trace $\mathrm{Tr}$ stands for the sum over all possible spin configurations. In writing Eq. (2), use is made of the identity

$$
\mathrm{e}^{\beta\left(\delta_{s_{i}, s_{j}}-1\right)}=(1-p)+p \delta_{s_{i}, s_{j}}
$$

with $p=1-\mathrm{e}^{-\beta}$, where here and in the sequel we set the coupling constant $K$ to unity. The identity (3) can be pictured as setting bonds with probability $p /[(1-p)+p]=p$ between two nearest neighbor spins in the same spin state for which $\delta_{s_{i}, s_{j}}=1$. When two nearest neighbor spins are not in the same spin state, $\delta_{s_{i}, s_{j}}=0$, then with probability $(1-p) /(1-p)=1$ the bond is not set, i.e., never. It thus follows, that the partition function can be equivalently written as

$$
Z_{\mathrm{FK}}=\sum_{\{\Gamma\}} p^{b}(1-p)^{\bar{b}+a} Q^{N_{\mathrm{C}}}
$$

where $\{\Gamma\}$ denotes the set of bond configurations realized on a total of $B$ bonds on the lattice. A given configuration is 
specified by $b$ set and $\bar{b}$ not set bonds between nearest neighbor spins in the same spin state, and $a$ pairs of nearest neighbor spins not in the same spin state (for which the bonds are never set). Together they add up to the total number of bonds, $B=b+\bar{b}+a$, so that the exponent $\bar{b}+a$ in Eq. (4) can also be written as $B-b$. Only spins connected by set bonds form a cluster. The exponent $N_{C}$ in Eq. (4) denotes the number of clusters, including isolated sites, contained in the bond configuration $\Gamma$. The factor $Q^{N_{\mathrm{C}}}$ arises because a given cluster can be in any of the $Q$ possible spin states. Equation (4) is the celebrated Fortuin-Kasteleyn (FK) representation of the Potts model [8]. It gives an equivalent representation of that spin model in terms of FK clusters obtained from the naive geometrical clusters of nearest neighbor spins in the same spin state, discussed in the Introduction, by putting bonds with a probability $p=1-\mathrm{e}^{-\beta}$ between nearest neighbors. As geometrical clusters are split up in the process, the resulting FK clusters are generally smaller and more loosely connected than the geometrical ones.

Not only does the FK representation provide a geometrical description of the phase transition in the Potts model, it also forms the basis of efficient Monte Carlo algorithms by Swendsen and Wang [10], and by Wolff [11], in which not individual spins are updated, but entire FK clusters. The main advantage of the nonlocal cluster update over a local spin update, like Metropolis or heat bath, is that it substantially reduces the critical slowing down near the critical point.

\section{B. FK Clusters}

The results of standard percolation theory [12] also apply to FK clusters. In particular, the distribution $\ell_{n}$ of FK clusters, giving the average number density of clusters of mass $n$, takes near the critical point the asymptotic form

$$
\ell_{n} \sim n^{-\tau} \mathrm{e}^{-\theta n} .
$$

The first factor, characterized by the exponent $\tau$, is an entropy factor, measuring the number of ways a cluster of mass $n$ can be embedded in the lattice. The second factor is a Boltzmann weight which suppresses large clusters when the parameter $\theta$ is finite. Clusters proliferate and percolate the lattice when $\theta$ tends to zero. The vanishing is characterized by a second exponent $\sigma$ defined via

$$
\theta \propto\left|T-T_{\mathrm{c}}\right|^{1 / \sigma}
$$

As in percolation theory [12], the values of the two exponents specifying the cluster distribution uniquely determine the critical exponents. To obtain these relations, we start by considering the radius of gyration $R_{n}$,

$$
R_{n}^{2}=\frac{1}{n} \sum_{i=1}^{n}\left(\mathbf{x}_{i}-\overline{\mathbf{x}}\right)^{2}=\frac{1}{2 n^{2}} \sum_{i, j=1}^{n}\left(\mathbf{x}_{i}-\mathbf{x}_{j}\right)^{2},
$$

with $\mathbf{x}_{i}$ the position vectors of the sites and $\overline{\mathbf{x}}=(1 / n) \sum_{i=1}^{n} \mathbf{x}_{i}$ the center of mass of the cluster. Asymptotically, the average $\left\langle R_{n}\right\rangle$ scales with the cluster mass $n$ as

$$
\left\langle R_{n}\right\rangle \sim n^{1 / D}
$$

which defines the Hausdorff, or fractal dimension $D$. The average radius of gyration $\left\langle R_{n}\right\rangle$ gives the typical linear size of a cluster of mass $n$. A second length scale is provided by the correlation length $\xi$, which diverges close to $T_{\mathrm{c}}$ with an exponent $v$ as $\xi \sim\left|T-T_{\mathrm{c}}\right|^{-v}$. Both are related via

$$
\left\langle R_{n}\right\rangle=\xi \mathrm{R}(n \theta),
$$

where $\mathrm{R}$ is a scaling function, cf. Eq. (5). From the asymptotic behavior (8), the divergence of the correlation length, and the vanishing (6) of the parameter $\theta$ as $T_{\mathrm{c}}$ is approached, the relation

$$
v=\frac{1}{\sigma D}
$$

follows, connecting the critical exponent $v$ to the fractal dimension $D$ of the clusters and $\sigma$.

The fractal dimension can also be related to the entropy exponent $\tau$ as follows. At criticality, the mass $n$ of a cluster is distributed over a volume of typical linear size $\left\langle R_{n}\right\rangle$, so that

$$
n \ell_{n} \sim 1 /\left\langle R_{n}\right\rangle^{d}
$$

with $d$ the dimension of the lattice. This leads to the wellknown expression

$$
\tau=\frac{d}{D}+1
$$

in terms of which the correlation length exponent reads $v=$ $(\tau-1) / d \sigma$.

\section{Improved Estimators}

To see how physical observables, such as the magnetization $m$ and the magnetic susceptibility $\chi$ are represented in terms of FK clusters, we consider the Ising model in the standard notation with the spin variable $S_{i}= \pm 1$ for simplicity. The correlation function $\left\langle S_{i} S_{j}\right\rangle$ has a particular simple representation. When the two spins belong to two different FK clusters

$$
\left\langle S_{i} S_{j}\right\rangle=\frac{1}{4} \sum_{S_{i}, S_{j}= \pm 1} S_{i} S_{j}=0
$$

while when they belong to the same cluster

$$
\left\langle S_{i} S_{j}\right\rangle=\frac{1}{2} \sum_{S_{i}=S_{j}= \pm 1} S_{i} S_{j}=1 .
$$

That is, if $C_{i}$ denotes the FK cluster to which the spin $S_{i}$ belongs and $C_{j}$ the one to which $S_{j}$ belongs, then

$$
\left\langle S_{i} S_{j}\right\rangle=\delta_{C_{i}, C_{j}}
$$

For the susceptibility $\chi \equiv \sum_{i j}\left\langle S_{i} S_{j}\right\rangle$ in the normal phase, Eq. (15) gives

$$
\chi=\sum_{i j} \delta_{C_{i}, C_{j}}=\sum_{\{C\}} n_{C}^{2}
$$


where the sum $\sum_{\{C\}}$ is over all FK clusters, and $n_{C}$ denotes the mass of a given cluster. In terms of the FK cluster distribution $\ell_{n}$, the susceptibility can be written as

$$
\chi=\sum_{n} n^{2} \ell_{n}
$$

Note that in percolation theory [12], the ratio $\sum_{n} n^{2} \ell_{n} / \sum_{n} n \ell_{n}$ denotes the average cluster size. Since in the Ising model all $L^{d}$ spins are part of some FK cluster, we have the constraint

$$
\sum_{n} n \ell_{n}=1 \text {. }
$$

It thus follows that the right hand of Eq. (17) precisely gives the average size of FK clusters. In other words, this geometrical observable directly measures the magnetic susceptibility of the Ising model. From the asymptotic form (5), and the divergence $\chi \sim\left|T-T_{\mathrm{c}}\right|^{-\gamma}$ of the susceptibility when the critical point is approached, the relation $\gamma=(3-\tau) / \sigma$ between the critical exponent $\gamma$ and the cluster exponents $\sigma$ and $\tau$ follows.

Also the magnetization $m$ has a simple geometrical representation [12]. In an applied magnetic field $H$, a spin cluster of mass $n_{C}$ has a probability $\propto \exp \left(\beta n_{C} H\right)$ to be oriented along the field direction, and a probability $\propto \exp \left(-\beta n_{C} H\right)$ to be oriented against the field direction. The difference between these probabilities gives the magnetization $m_{C}$ per spin in the cluster,

$$
m_{C}=\tanh \left(\beta n_{C} H\right) .
$$

Close to the critical temperature and in the thermodynamic limit $L \rightarrow \infty$, the largest cluster dominates, and $\tanh \left(\beta n_{\max } H\right) \rightarrow \pm 1$ for this cluster, depending on its orientation. The magnetization of the entire system (per spin) then becomes

$$
m= \pm P_{\infty},
$$

where $P_{\infty}=n_{\max } / L^{d}$ gives the fraction of spins in the largest cluster-the so-called percolation strength. Because of the constraint (18), it is related to the FK cluster distribution via

$$
P_{\infty}=1-\sum_{n}^{\prime} n \ell_{n}
$$

where the prime on the sum indicates that the largest FK spin cluster is to be excluded. The magnetization vanishes near the critical point as $m \sim\left|T-T_{\mathrm{c}}\right|^{\beta}$. Together with the asymptotic behavior of the cluster distribution, Eq. (20) with Eq. (21) gives the relation $\beta=(\tau-2) / \sigma$.

These geometrical observables (average cluster size and percolation strength) are called improved estimators because they usually have a smaller standard deviation than the spin observables.

The results just derived for the Ising model also apply to the rest of the critical Potts models [8]. In this way, the thermal critical exponents of these models are completely determined by the exponents $\sigma$ and $\tau$, characterizing the FK cluster distribution. Specifically,

$$
\begin{array}{lll}
\alpha=2-\frac{\tau-1}{\sigma}, & \beta=\frac{\tau-2}{\sigma}, & \gamma=\frac{3-\tau}{\sigma}, \\
\eta=2+d \frac{\tau-3}{\tau-1}, & v=\frac{\tau-1}{d \sigma},
\end{array}
$$

as in percolation theory [12]. The exponent $\eta$, determining the algebraic decay of the correlation function at the critical point, is related to the fractal dimension via

$$
D=\frac{1}{2}(d+2-\eta)
$$

Consequently

$$
\gamma / \mathrm{v}=2 D-d
$$

\section{Critical Exponents}

The critical exponents of the 2D $Q$-state Potts model are known exactly [13]. It is convenient to parametrize the models as

$$
\sqrt{Q}=-2 \cos (\pi / \bar{\kappa}),
$$

with $2 \geq \bar{\kappa} \geq 1$. For the Ising model $(Q=2) \bar{\kappa}=4 / 3$, while for uncorrelated percolation $(Q \rightarrow 1) \bar{\kappa}=3 / 2$. The correlation length exponent $v$ and the exponent $\eta$ are given in this representation by [13]:

$$
\frac{1}{\mathrm{v}}=y_{\mathrm{T}, 1}=3-\frac{3}{2} \bar{\kappa}, \quad \eta=2-\frac{1}{\bar{\kappa}}-\frac{3}{4} \bar{\kappa},
$$

where $y_{\mathrm{T}, 1}$ is the leading thermal exponent. The next-toleading thermal exponent $y_{\mathrm{T}, 2}$ reads $y_{\mathrm{T}, 2}=4(1-\bar{\kappa})$, which is negative for $\bar{\kappa} \geq 1$, implying that the corresponding operator is an irrelevant perturbation. The other critical exponents can be obtained through standard scaling relations. The parameter $\overline{\mathrm{K}}$ is related to the central charge $c$, defining the universality class, via [14]

$$
c=1-\frac{6(1-\overline{\mathrm{\kappa}})^{2}}{\overline{\mathrm{K}}} .
$$

Finally, the fractal dimension $D$ of FK clusters is given by $[15,16]$

$$
D=1+\frac{1}{2 \bar{\kappa}}+\frac{3}{8} \bar{\kappa}
$$

which gives $D=15 / 8$ for the Ising model and $D=91 / 48$ for uncorrelated percolation.

To demonstrate that FK clusters actually percolate at the critical point, Fig. 3 shows the distribution $\ell_{n}$ of these clusters in the $2 \mathrm{D}$ Ising model at criticality $(\theta=0)$ on a square lattice of linear size $L=512$. With $D=15 / 8$, it follows from Eq. (12) that the entropy exponent takes the value $\tau=31 / 15$. The straight line, obtained through a one-parameter fit with the slope fixed to the predicted value, shows that asymptotically the FK cluster distribution has the expected behavior.

\section{E. Swendsen-Wang Cluster Update}

The theoretical predictions (22) can be directly verified through Monte Carlo simulations, using the Swendsen-Wang 


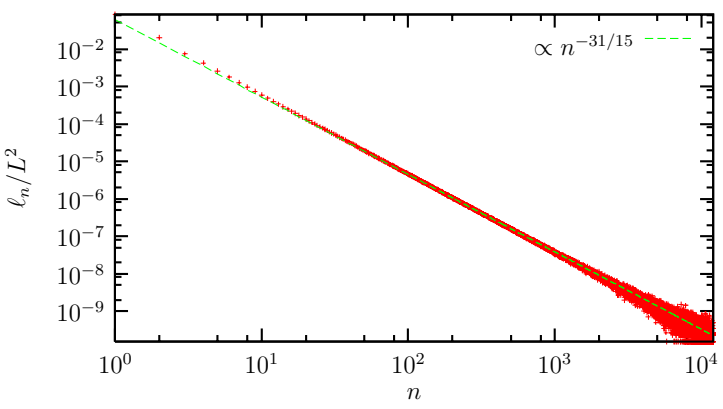

FIG. 3: Distribution $\ell_{n}$ normalized to the volume $L^{2}$ of FK clusters in the $2 \mathrm{D}$ Ising model at criticality on a square lattice of linear size $L=512$. Statistical error bars are omitted from the data points for clarity. The straight line is a one-parameter fit through the data points with (minus) the slope fixed to the predicted value $\tau=31 / 15=2.06666 \cdots$. The fit illustrates that asymptotically the distribution is algebraic, as expected at criticality.

cluster update [10]. Instead of single spins, entire FK clusters are considered units to be flipped as a whole in this approach. Standard finite-size scaling theory applied to the percolation strength $P_{\infty}$ and the average cluster size $\chi$ gives the scaling laws

$$
P_{\infty}=L^{-\beta / v} \mathrm{P}(L / \xi), \quad \chi=L^{\gamma / v} \times(L / \xi),
$$

with $\mathrm{P}$ and $\mathrm{X}$ scaling functions. Precisely at $T_{\mathrm{c}}$, these scaling relations imply an algebraic dependence on the system size $L$, allowing for a determination of the exponent ratios $\beta / v$ (see Fig. 4) and $\gamma / \nu$. Using these geometrical observables as improved estimators for the magnetization and susceptibility, respectively, we arrived at the estimates for the Ising model $(Q=2)[17]$

$$
\begin{aligned}
& \beta / \nu=0.1248(8) \approx 1 / 8, \\
& \gamma / \nu=1.7505(12) \approx 7 / 4,
\end{aligned}
$$

where the right hands give the known values for the Ising critical exponents. These estimates illustrate first of all that FK clusters indeed encode the thermal critical behavior of the Ising model. Moreover, they also illustrate that measuring geometrical observables gives excellent results for the critical exponents. The data were fitted over the range $L=64-512$, using the least-squares Marquardt-Levenberg algorithm.

In Ref. [18], the fractal dimension of FK clusters were obtained from analyzing their distribution. This method gives less accurate results than applying finite-size scaling to improved estimators. The main problem is related to the fitting window. The fitting range cannot be started at too small cluster sizes, where the distribution has not taken on its asymptotic form yet, while too large cluster sizes, which are generated only a few times during a complete Monte Carlo run, are also to be excluded because of the noise in the data and finite-size effects. The results depend sensitively on the precise choice of the fitting window.

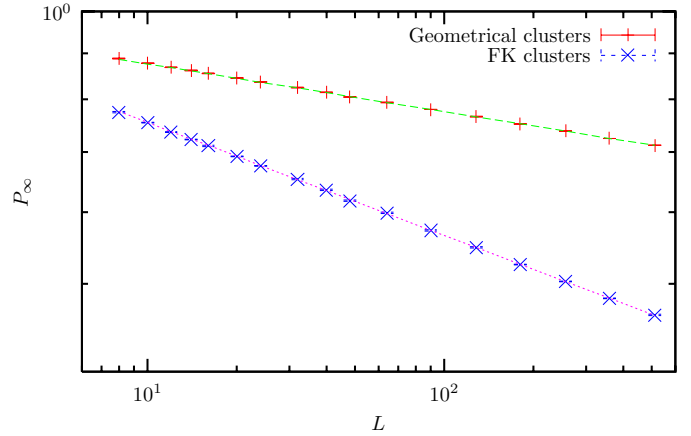

FIG. 4: Log-log plot of the percolation strength $P_{\infty}$ of geometrical and FK clusters at criticality in the 2D Ising model as a function of the linear system size $L$. The straight lines $0.988281 L^{-0.0527}$ for geometrical and $1.00558 L^{-0.1248}$ for FK clusters are obtained from two-parameter fits through the data points. Statistical error bars are smaller than the symbol sizes.

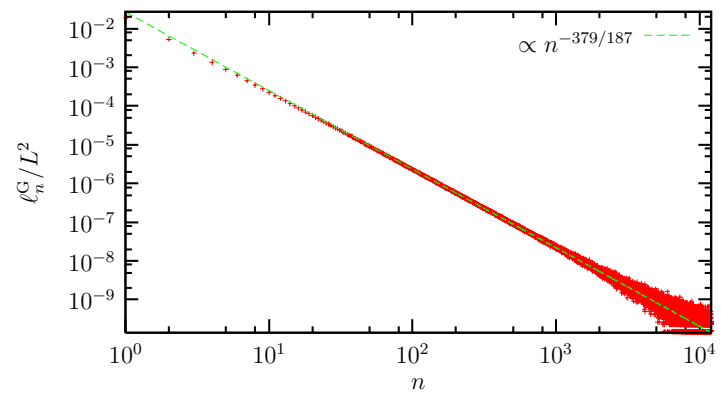

FIG. 5: Distribution $\ell_{n}^{\mathrm{G}}$ normalized to the volume $L^{2}$ of geometrical clusters in the 2D Ising model at criticality on a square lattice of linear size $L=512$. Statistical error bars are omitted from the data points for clarity. The straight line is a one-parameter fit through the data points with (minus) the slope fixed to the value $\tau^{\mathrm{G}}=379 / 187=$ $2.02673 \cdots$. The fit illustrates that asymptotically the distribution is algebraic at criticality.

\section{F. Geometrical Clusters}

Figure 2 suggests that the geometrical spin clusters also percolate right at the Curie point of the Ising model. To demonstrate this to be the case, Fig. 5 shows the distribution of these clusters at criticality. Asymptotically, the distribution indeed shows algebraic behavior, implying that clusters of all size appear in the system. It is therefore natural to investigate the exponents associated with the percolation strength $P_{\infty}^{\mathrm{G}}$ (see Fig. 4) and the average size $\chi^{\mathrm{G}}$ of these geometrical clusters (the superscript "G" refers to geometrical clusters). Using finite-size scaling, as for the FK clusters, we arrived at the estimates [17]

$$
\begin{aligned}
& \beta^{\mathrm{G}} / \nu=0.0527(4) \approx 5 / 96=0.0520 \cdots, \\
& \gamma^{\mathrm{G}} / \nu=1.8951(5) \approx 91 / 48=1.8958 \cdots .
\end{aligned}
$$


In obtaining these estimates we included percolating clusters. When excluding them, as was done in Ref. [19], the estimates become less accurate [17]. The entropy exponent which follows from these results is $\tau^{\mathrm{G}}=379 / 187$, corresponding to the fractal dimension $D^{\mathrm{G}}=187 / 96$.

It should be stressed that only in $2 \mathrm{D}$ geometrical clusters percolate right at the critical temperature. In higher dimensions, geometrical clusters percolate in general too early at a lower temperature, and their fractal structure is unrelated to any thermodynamic singularity.

The 2D exponents (31) are not related to the critical behavior of the Ising model and the question arises: What do these exponents describe?

\section{TRICRITICAL POTTS MODEL}

\section{A. Dual Map}

When the pure 2D Potts model is extended to include vacant sites, it displays in addition to critical also tricritical behavior at the same critical temperature $T_{\mathrm{c}}$ [20]. The tricritical behavior is known to be intimately connected to the critical behavior, and both critical points share the same central charge. To demonstrate this connection, note that for a given $c$, Eq. (27) yields two solutions for $\bar{\kappa}$ :

$$
\bar{\kappa}_{ \pm}=\frac{13-c \pm \sqrt{(c-25)(c-1)}}{12}
$$

with $\bar{\kappa}_{+} \bar{\kappa}_{-}=1$, where $\bar{\kappa} \equiv \bar{\kappa}_{+} \geq 1$ and hence $\bar{\kappa}_{-} \leq 1$. Stated alternatively, the substitution $\overline{\bar{K}}$ with $1 / \bar{\kappa}$ leaves the central charge (27) unchanged, $c(\overline{\mathrm{K}})=c(1 / \overline{\mathrm{K}})$. When applied to the parametrization (25) of the critical Potts branch, this so-called dual map yields the parametrization [14]

$$
\sqrt{Q^{\mathrm{t}}}=-2 \cos (\pi \bar{\kappa})
$$

of the tricritical branch (the superscript " $\mathrm{t}$ " refers to the tricritical point). Various results for the critical point [13] can be simply transcribed to the tricritical point by using this dual map, leading to $[20,21]$

$$
\frac{1}{\mathrm{v}^{\mathrm{t}}}=y_{\mathrm{T}, 1}^{\mathrm{t}}=3-\frac{3}{2 \overline{\mathrm{\kappa}}}, \quad \eta^{\mathrm{t}}=2-\bar{\kappa}-\frac{3}{4 \bar{\kappa}}
$$

while the next-to-leading thermal exponent becomes

$$
y_{\mathrm{T}, 2}^{\mathrm{t}}=4-\frac{4}{\overline{\mathrm{\kappa}}} \text {. }
$$

To preserve relation (23) under the dual map, the fractal dimensions of the geometrical and FK clusters must also be related by the map $\bar{\kappa} \rightarrow 1 / \bar{\kappa}[22,23]$. This gives

$$
D^{\mathrm{G}}=1+\frac{\bar{\kappa}}{2}+\frac{3}{8 \bar{\kappa}},
$$

which is indeed the correct fractal dimension of geometrical clusters $[24,25]$. In other words, the geometrical clusters can, as far as their scaling behavior is concerned, be considered shadows of the FK clusters. The use of the word "shadow" will become clear when we consider the cluster boundaries in the next section.

\section{B. Ising \& its $Q^{\mathrm{t}}=1$ Potts Shadow}

Equation (36) gives as fractal dimension of the geometrical clusters of the Ising model $(\bar{\kappa}=4 / 3) D^{\mathrm{G}}=187 / 96$, implying via Eq. (12) $\tau=379 / 187$, in accordance with what we found numerically [17]. Note that with $\bar{\kappa}=4 / 3$, Eq. (33) gives $Q^{t}=$ 1. That is, the tricritical model described by the geometrical clusters of the Ising model is the diluted $Q^{\mathrm{t}}=1$ Potts model. Both models share the same central charge $c=1 / 2$.

The alert reader may have noticed a curiosity concerning the thermal exponents. According to Eq. (34), the correlation length exponent $v^{t}$ takes the value $v^{t}=1 / y_{T, 1}^{t}=8 / 15$ in the diluted $Q^{t}=1$ Potts model $(\bar{\kappa}=4 / 3)$. Yet, in our numerical investigation [17] of the geometrical clusters of the Ising model, we seem to observe the correlation length exponent $v=1$ of the Ising model. Hence, $v$ and not the tricritical exponent $v^{t}$ appears in Eq. (31). In fact, what we see is the tricritical nextto-leading thermal exponent (35), which for the diluted $Q^{\mathrm{t}}=1$ Potts model happens to take the same value as the leading thermal exponent of the Ising model, $y_{\mathrm{T}, 2}^{\mathrm{t}}=y_{\mathrm{T}, 1}=1$ for $\bar{\kappa}=4 / 3$.

\section{HULLS \& EXTERNAL PERIMETERS}

\section{A. FK Clusters}

When clusters percolate at a certain threshold, their boundaries necessarily do too. In the context of uncorrelated percolation in $2 \mathrm{D}$, external cluster boundaries can be traced out by a biased random walker as follows [26]. The algorithm starts by identifying two endpoints on a given cluster, and putting the random walker at the lower endpoint. The walker is instructed to first attempt to move to its nearest neighbor to the left. If that site is vacant, the walker should try to move straight ahead. If that site is also vacant, the walker should try to move to its right. Finally, if also that site is vacant, the walker is instructed to return to the previous site, to discard the direction already explored, and to investigate the (at most two) remaining directions in the same order. When turning left or right, the walker changes its orientation accordingly. The procedure is repeated iteratively until the upper endpoint is reached. The other half of the boundary is obtained by repeating the entire algorithm for a random walker instructed to first attempt to move to its right rather than to its left.

For FK clusters, being built from bonds between nearest neighbor sites with their spin in the same spin state, one can imagine two different external boundaries (see Fig. 6). First, one can allow the random walker to move along the FK boundary only via set bonds. This defines the hull of the cluster. Second, one can allow the random walker to move to a nearest neighbor site on the FK boundary irrespective of whether the bond is set or not. This defines the external perimeter of the cluster, which is a smoother version of the hull.

Since boundaries are clusters themselves, they too are characterized by a fractal dimension and a distribution like Eq. (5) specified by two exponents $\sigma$ and $\tau$. 

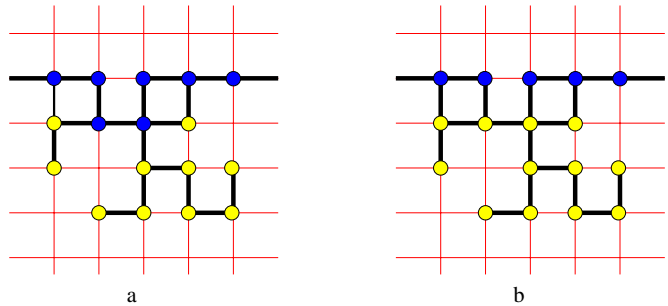

FIG. 6: In both panels, a piece of the same single FK cluster of nearest neighbor sites (filled circles) connected by bonds (black links) is shown. Two different external boundaries can be defined: (a) The hull (dark filled circles) is found by allowing a random walker tracing out the boundary to move only over set bonds. (b) The external perimeter (dark filled circles) is found by allowing the random walker to move to a nearest neighbor on the cluster boundary irrespective of whether the connecting bond is set or not. The external perimeter, which contains two sites less than the hull for this boundary segment, is therefore a smoother version of the hull.

\section{B. Fractal Dimensions}

The fractal dimensions of the hulls $(\mathrm{H})$ and external perimeters (EP) of FK clusters are given by [16, 27, 28]

$$
D_{\mathrm{H}}=1+\frac{\bar{\kappa}}{2}, \quad D_{\mathrm{EP}}=1+\frac{1}{2 \bar{\kappa}}
$$

As for clusters, the average hull and external perimeter sizes diverge at the percolation threshold. Let $\gamma_{\mathrm{H}}$ and $\gamma_{\mathrm{EP}}$ denote the corresponding exponents, then because of Eq. (24) with $d=2$ and Eq. (37)

$$
\gamma_{\mathrm{H}} / \nu=\bar{\kappa}, \quad \gamma_{\mathrm{EP}} / \nu=1 / \bar{\kappa}
$$

where a single correlation length exponent $v$ is assumed.

For illustrating purposes, Fig. 7 shows the distribution of the two boundaries of FK clusters in the Ising model at criticality. The straight lines are one-parameter fits through the data points with the slopes fixed to the expected values. Although the estimates for $D_{\mathrm{H}}$ and $D_{\mathrm{EP}}$ we obtained, using finite-size scaling applied to the improved estimators at criticality, are compatible with the theoretical conjectures [17], the achieved precision is less than the one we reached for the clusters themselves. The reason for this is as follows. While including percolating clusters when considering the mass of the clusters, we ignore them in tracing out cluster boundaries. Because of the finite lattice size, large percolating clusters have anomalous small (external) boundaries, so that including them would distort the boundary distributions. Moreover, the Grossman-Aharony algorithm [26] used to trace out cluster boundaries generally fails on a percolating cluster as its boundary not necessarily forms a single closed loop any longer. However, as we explicitly demonstrated for the cluster mass [17], disregarding percolating clusters leads to strong corrections to scaling, and therefore to less accurate results.

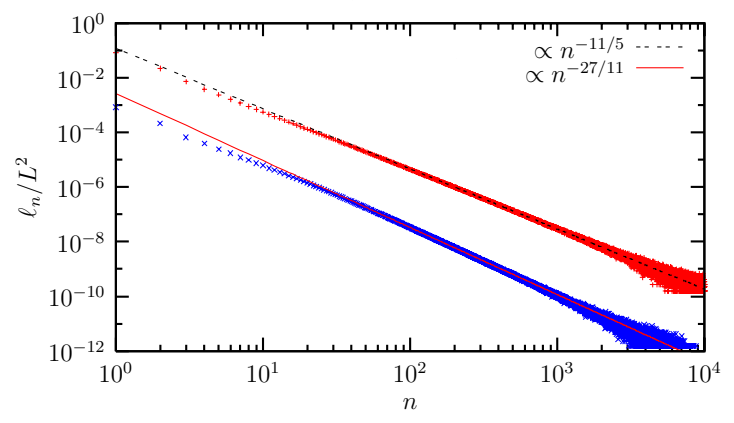

FIG. 7: Distribution normalized to the volume $L^{2}$ of the hulls and external perimeters of FK clusters in the 2D Ising model at criticality on a square lattice of linear size $L=512$. Statistical error bars are omitted from the data points for clarity. The straight lines are oneparameter fits through the data points with the slopes fixed to the expected values. For clarity, the external perimeters distribution is shifted downward by two decades.

\section{Geometrical Clusters}

For geometrical clusters, where the bond between nearest neighbor sites with their spin in the same spin state is so to speak always set, hulls and external perimeters cannot be distinguished, and

$$
D_{\mathrm{H}}^{\mathrm{G}}=D_{\mathrm{EP}}^{\mathrm{G}} .
$$

The fractal dimension of the boundary is gotten from that of the hull (37) of FK clusters by applying the dual map $\bar{\kappa} \rightarrow 1 / \bar{\kappa}$, yielding [29]

$$
D_{\mathrm{H}}^{\mathrm{G}}=1+\frac{1}{2 \bar{\kappa}} \text {. }
$$

Since FK clusters have two boundaries, while geometrical clusters have only one, geometrical clusters have less structure and can be considered shadows of FK clusters under the dual map, as far as their scaling behavior is concerned.

Again for illustrating purposes, Fig. 8 shows the distribution of the hulls of geometrical clusters in the Ising model at criticality. The slow approach to the asymptotic form, with the associated strong corrections to scaling we observed for the hulls of geometrical clusters, stands out clearly from the other distributions. The reason for this is that geometrical clusters have a larger extent than FK clusters. On a finite lattice, percolating clusters gulp up smaller ones reached by crossing lattice boundaries. For geometrical clusters this happens more often than for FK clusters, so that disregarding percolating clusters when tracing out cluster boundaries has a more profound effect. In particular, the average hull size is underestimated. With increasing lattice size, the effect becomes smaller, as we checked explicitly [17].

This behavior is different from what we found in another numerical study of the hulls of geometrical clusters in the $2 \mathrm{D}$ Ising model [22]. In that Monte Carlo study, we used a plaquette update to directly simulate the hulls. Although the largest 


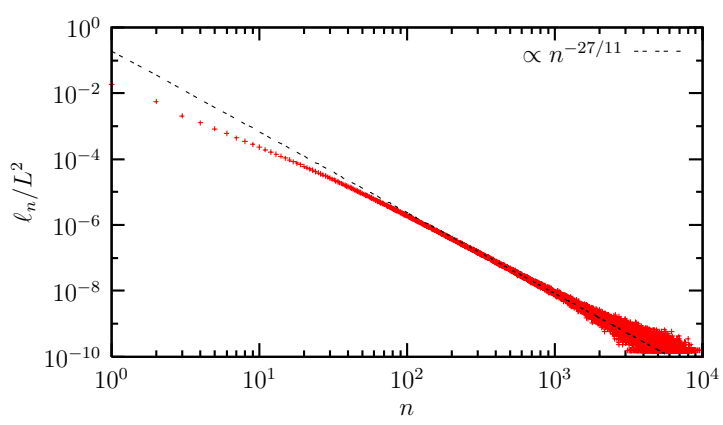

FIG. 8: Distribution normalized to the volume $L^{2}$ of the hulls of geometrical clusters in the 2D Ising model at criticality on a square lattice of linear size $L=512$. Statistical error bars are omitted from the data points for clarity. The straight line is a one-parameter fit through the data points with the slope fixed to the expected value.

hull was omitted in each measurement, we found corrections to scaling to be virtually absent (see Fig. 11 of that paper). This allowed us to obtain a precise estimate for the fractal dimension on relatively small lattices.

\section{CONCLUSIONS \& OUTLOOK}

As illustrated in these notes, for the 2D Potts models it is well established that suitably defined geometrical objects encode in their fractal structure critical behavior. In fact, two types of spin clusters exist, viz., FK and geometrical clusters, which both proliferate precisely at the thermal critical point. As emphasized before, this is special to 2D. In general, geometrical clusters percolate at an inverse temperature $\beta_{\mathrm{p}}>\beta_{\mathrm{c}}$. The fractal structure of FK clusters encodes the critical exponents of the critical Potts model, while that of geometrical clusters in $2 \mathrm{D}$ encodes those of the tricritical Potts model. The fractal structure of the two cluster types as well as the two fixed points are closely related, being connected by the dual map $\bar{\kappa} \rightarrow 1 / \bar{\kappa}$. This map conserves the central charge, so that both fixed points share the same central charge. The geometrical clusters can, as far as scaling properties are concerned, be considered shadows of the FK clusters.

Up to now we considered external boundaries of spin clusters as clusters themselves, which necessarily percolate when the spin clusters do. An alternative way of looking at these boundaries is to consider them as loops. In this approach, it is natural to extend the Ising model in another way and to consider the $\mathrm{O}(N)$ spin models, with $-2 \leq N \leq 2$. The high-temperature (HT) representation of the critical $\mathrm{O}(N)$ spin model naturally defines a loop gas, corresponding to a diagrammatic expansion of the partition function in terms of closed graphs along the bonds on the underlying lattice [30]. The loops percolate right at the critical temperature, and similar arguments as given in these notes for spin clusters show that the fractal structure of these geometrical objects encode important information concerning the thermal critical $\mathrm{O}(N)$ behavior [31, 32]. This connection was first established by de Gennes [33] for self-avoiding walks, which are described by the $\mathrm{O}(N)$ model in the limit $N \rightarrow 0$. One aspect in which lines differ from spin clusters is that they can be open or closed. It is well known from the work on self-avoiding walks that the loop distribution itself is not sufficient to establish the critical behavior, as has recently also been emphasized in Ref. [34]. For this, also the total number $z_{n} \equiv \sum_{j} z_{n}\left(\mathbf{x}_{i}, \mathbf{x}_{j}\right)$ of open graphs of $n$ steps starting at $\mathbf{x}_{i}$ and ending at an arbitrary site $\mathbf{x}_{j}$ is needed. Its asymptotic behavior close to the critical temperature, cf. Eq. (5) with Eq. (12),

$$
z_{n} \sim n^{\vartheta / D} \mathrm{e}^{-\theta n}
$$

provides an additional exponent $\vartheta$, which together with the loop distribution exponents is needed to specify the full set of critical exponents [32]. In Eq. (41), $D$ denotes the fractal dimension of the closed graphs. Note that for spin clusters, the notion of open or closed does not apply, so that the analog of the exponent $\vartheta$ is absent there.

Remarkably, the HT graphs of a given critical $\mathrm{O}(N)$ model represent at the same time the hulls of the geometrical clusters in the $Q$-state Potts model with the same central charge $[22,25,35]$. To close the circle, we note that, as in the Potts model, including vacancies in the $\mathrm{O}(N)$ model gives rise to also tricritical behavior. The tricritical point corresponds to the point where the HT graphs collapse. In the context of selfavoiding walks $(N \rightarrow 0)$, this point is known as the $\Theta$ point. Using the duality discussed in Sec. III A, we recently conjectured that the tricritical HT graphs at the same time represent the hulls of the FK clusters of the Potts model with the same central charge $c$ as the tricritical $\mathrm{O}(N)$ model [31]. This connection allowed us to predict the magnetic scaling dimension of the $\mathrm{O}(N)$ tricritical model, in excellent agreement with recent high-precision Monte Carlo data in the range $0 \leq c \lesssim 0.7$ [36].

We started these notes mentioning the $\lambda$ transition in liquid ${ }^{4} \mathrm{He}$ in terms of vortex proliferation. In closing, we wish to give the present status of that picture as established in a very recent high-precision Monte Carlo study of the 3D complex $|\phi|^{4}$ theory describing the transition [37]. An important observable is the total vortex line density $v$. By means of standard finite-size scaling analysis of the associated susceptibility $\chi=L^{3}\left(\left\langle v^{2}\right\rangle-\langle v\rangle^{2}\right)$, the inverse critical temperature $\beta_{\mathrm{c}}$ was estimated and shown to be perfectly consistent with the estimate of a previous study directly in terms of the original variables [38]. Unfortunately, when considering percolation observables, such as whether a vortex loop percolates the lattice, slight but statistically significant deviations from $\beta_{c}$ were found. For all observables considered, the percolation threshold $\beta_{\mathrm{p}}>\beta_{\mathrm{c}}$. That is, from these observables one would conclude that the vortices proliferate too early at a temperature below the critical one (as do geometrical clusters in 3D). Yet, when taking the percolation threshold as an adjustable parameter, reasonable estimates were obtained from percolation observables for the critical exponents $v$ and $\beta$, consistent with those of the XY model. The problem with the percolation threshold is quite possibly related to the way vortex loops are 
traced out. When two vortex segments enter a unit cell, it is not clear how to connect them with the two outgoing segments. A popular choice is to randomly connect them, but it might well be that the resulting network is too extended and consequently percolates too early. It is in our mind conceivable that a proper prescription for connecting vortex segments could lead to a vortex percolation threshold right at the critical temperature, in the spirit of the FK construction.

As a final remark, we note that even in cases where no thermodynamic phase transition takes place, the notion of vortex proliferation can be useful in understanding the phase structure of the system under consideration. An example is provided by the $3 \mathrm{D}$ Abelian Higgs lattice model with compact gauge field [39]. In addition to vortices, the compact model also features magnetic monopoles as topological defects. It is well established that in the London limit, where the amplitude of the Higgs field is frozen, it is always possible to move from the Higgs region into the confined region without encountering thermodynamic singularities [40]. Nevertheless, the susceptibility data for various observables define a precisely located phase boundary. Namely, for sufficiently large lattices, the maxima of the susceptibilities at the phase bound- ary do not show any finite-size scaling. Moreover, the susceptibility data obtained on different lattice sizes collapse onto single curves without rescaling, indicating that the infinitevolume limit is reached. In Ref. [39] it was argued that this phase boundary marks the location where the vortices proliferate. A well-defined and precisely located phase boundary across which geometrical objects proliferate, yet thermodynamic quantities remain nonsingular has become known as a Kertész line. Such a line was first introduced in the context of the Ising model in the presence of an applied magnetic field [41].

\section{Acknowledgment}

W. J. would like to thank the organisers of this conference for their warm hospitality. A. S. is indebted to Professor H. Kleinert for the kind hospitality at the Freie Universität Berlin. This work was partially supported by the Deutsche Forschungsgemeinschaft (DFG) under grant No. JA 483/173 and the EU RTN-Network 'ENRAGE': Random Geometry and Random Matrices: From Quantum Gravity to Econophysics under grant No. MRTN-CT-2004-005616.
[1] L. Onsager, Nuovo Cimento Suppl. 6, 249 (1949).

[2] R. P. Feynman, in: Progress in Low Temperature Physics, edited by C. J. Gorter (North-Holland, Amsterdam, 1955), Vol. 1, p. 17.

[3] R. P. Feynman, Phys. Rev. 90, 1116 (1953); ibid. 91, 1291 (1953); Statistical Mechanics (Benjamin, Reading, 1972).

[4] S. Bund and A. M. J. Schakel, Mod. Phys. Lett. B 13, 349 (1999).

[5] A. M. J. Schakel, Phys. Rev. E 63, 026115 (2001).

[6] D. M. Ceperley and E. L. Pollock, Phys. Rev. Lett. 56, 351 (1986).

[7] D. M. Ceperley, Rev. Mod. Phys. 67, 279 (1995)

[8] C. M. Fortuin and P. W. Kasteleyn, Physica 57, 536 (1972).

[9] R. B. Potts, Proc. Camb. Phil. Soc. 48, 106 (1952).

[10] R. H. Swendsen and J. S. Wang, Phys. Rev. Lett. 58, 86 (1987).

[11] U. Wolff, Phys. Rev. Lett. 62, 361 (1989).

[12] D. Stauffer and A. Aharony, Introduction to Percolation Theory, 2nd edition (Taylor \& Francis, London, 1994).

[13] M. P. M. den Nijs, J. Phys. A 12, 1857 (1979); Phys. Rev. B 27, 1674 (1983).

[14] J. Cardy, in: Phase Transitions and Critical Phenomena, edited by C. Domb and J. L. Lebowitz (Academic, London, 1987), Vol. 11, p. 55.

[15] H. E. Stanley, J. Phys. A 10, L211 (1977).

[16] A. Coniglio, Phys. Rev. Lett. 62, 3054 (1989).

[17] W. Janke and A. M. J. Schakel, Phys. Rev. E 71, 036703 (2005).

[18] J. Asikainen, A. Aharony, B. B. Mandelbrot, E. M. Rauch, and J.-P. Hovi, Eur. Phys. J. B 34, 479 (2003).

[19] S. Fortunato, Phys. Rev. B 66, 054107 (2002).

[20] B. Nienhuis, A. N. Berker, E. K. Riedel, and M. Schick, Phys. Rev. Lett. 43, 737 (1979).

[21] B. Nienhuis, J. Phys. A 15, 199 (1982); in: Phase Transitions and Critical Phenomena, edited by C. Domb and J. L. Lebowitz
(Academic, London, 1987), Vol. 11, p. 1.

[22] W. Janke and A. M. J. Schakel, Nucl. Phys. B [FS] 700, 385 (2004).

[23] Y. Deng, H. W. J. Blöte, and B. Nienhuis, Phys. Rev. E 69, 026123 (2004).

[24] A. L. Stella and C. Vanderzande, Phys. Rev. Lett. 62, 1067 (1989).

[25] B. Duplantier and H. Saleur, Phys. Rev. Lett. 63, 2536 (1989).

[26] T. Grossman and A. Aharony, J. Phys. A 19, L745 (1986).

[27] H. Saleur and B. Duplantier, Phys. Rev. Lett. 58, 2325 (1987).

[28] B. Duplantier, Phys. Rev. Lett. 84, 1363 (2000).

[29] C. Vanderzande, J. Phys. A 25, L75 (1992).

[30] H. E. Stanley, Introduction to Phase Transitions and Critical Phenomena (Oxford University Press, New York, 1971).

[31] W. Janke and A. M. J. Schakel, Phys. Rev. Lett. 95, 135702 (2005).

[32] W. Janke and A. M. J. Schakel, Anomalous scaling and fractal dimensions, cond-mat/0508734 (2005).

[33] P. G. de Gennes, Phys. Lett. A 38, 339 (1972).

[34] N. Prokof'ev and B. Svistunov, cond-mat/0504008 (2005).

[35] C. Vanderzande and A. L. Stella, J. Phys. A 22, L445 (1989).

[36] W. Guo, H. W. J. Blöte, and Y.-Y. Liu, Commun. Theor. Phys. (Beijing) 41, 911 (2004).

[37] E. Bittner, A. Krinner, and W. Janke, Phys. Rev. B 72, 094511 (2005).

[38] E. Bittner and W. Janke, Phys. Rev. Lett. 89, 130201 (2002); Phys. Rev. B 71, 024512 (2005).

[39] S. Wenzel, E. Bittner, W. Janke, A. M. J. Schakel, and A. Schiller, Phys. Rev. Lett. 95, 051601 (2005).

[40] E. Fradkin and S. H. Shenker, Phys. Rev. D 19, 3682 (1979).

[41] J. Kertész, Physica A 161, 58 (1989). 